\section{Periampullary carcinoid tumor}

Screening upper endoscopy in an asymptomatic 71-year-old man revealed a protruding periampullary tumor that was covered with intact duodenal mucosa (Figure 1). Biopsy specimens from this lesion revealed carcinoid tumor. Endoscopic ultrasound demonstrated an echopoor mass, $15 \mathrm{~mm}$ in diameter, in the mucosa. Abdominal computed tomography and magnetic resonance imaging studies showed that there were no metastases to the lymph nodes or to the liver. Endoscopic retrograde cholangiopancreatography was performed and this showed that there was no extension of the tumor into the common bile duct or into the pancreatic duct. The tumor was elevated by submucosal injection of hypertonic salineepinephrine, and an endoscopic mucosal resection was then successfully performed with no complications.

Macroscopically, the lesion consisted of a discrete mass within the mucosal layer that was found to be composed of multiple nests of small cells (Figure 2 ). Microscopically, these cells were found to consist of an eosinophilic cytoplasm, and uniform, oval, hyperchromatic nuclei that showed no atypia or mitotic activity. Immunohistochemically, the tumor cells were diffusely positive for somatostatin, but negative for insulin and gastrin. Based on these histological findings, we finally diagnosed this lesion as a carcinoid tumor (a well-differentiated endocrine tumor). The resection margins were all tumorfree. Surveillance duodenoscopy at 6 months revealed no macroscopic or microscopic evidence of tumor recurrence (Figure 3).

Previous studies of patients with ampullary carcinoids have reported that tumor size has no prognostic implications; in addition, no correlation could be identified between mitotic activity and metastatic potential $[1-3]$. The tumors that were included in the carcinoid group were predominantly well-differentiated endocrine tumors, and the prognosis was reported to be excellent, with an overall 5-year sur-

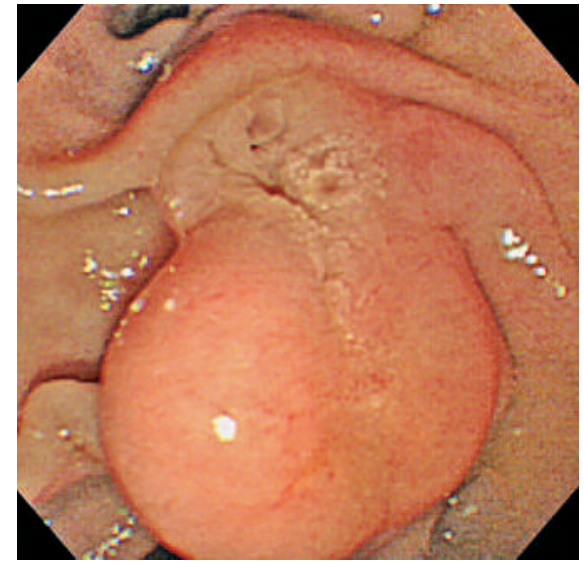

Figure 1 Endoscopic examination revealed a protruding periampullary tumor covered with intact duodenal mucosa.

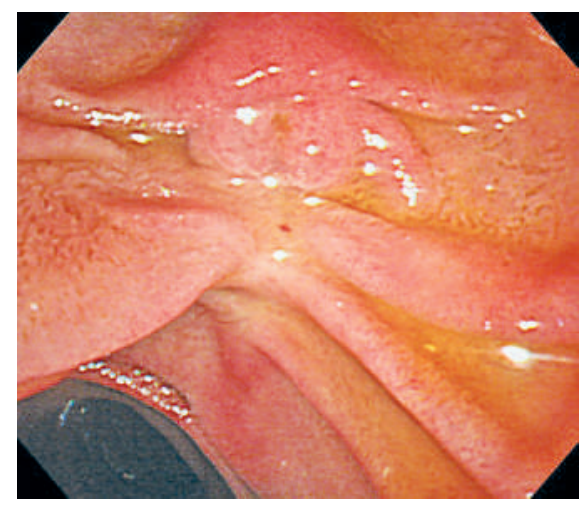

Figure 3 Surveillance duodenoscopy 6 months after resection of the carcinoid tumor revealed no macroscopic evidence of tumor recurrence.

vival rate of $90 \%[1,3]$. Local resection showed satisfactory results in tumors measuring less than $2 \mathrm{~cm}[3,4]$. Although the method of treatment for ampullary carcinoid tumors with a diameter of less than $2 \mathrm{~cm}$, no invasion of the muscularis propria, and no evidence of metastases remains controversial, endoscopic resection could be considered as a possible treatment modality as long as a strict follow-up protocol is adhered to, as in our case.

Endoscopy_UCTN_Code_CCL_1AB_2AZ_3AB

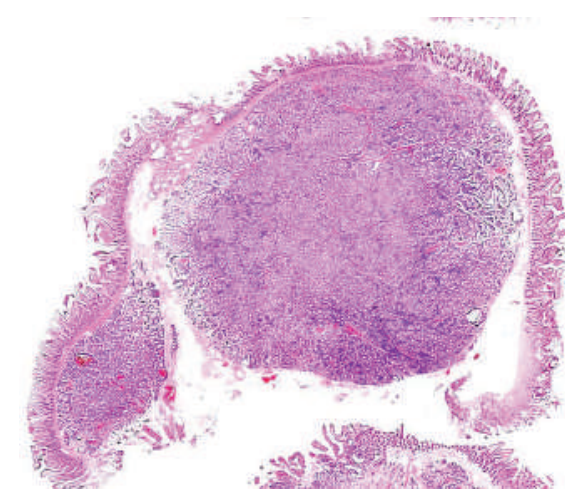

Figure 2 Specimens from the endoscopic resection revealed a discrete mass within the mucosal layer, composed of multiple nests of small cells.

\section{H. Fukatsu, H. Kawamoto, M. Fujii, K. Tsutsumi, H. Kato, K. Hirao, N. Kurihara, Y. Okamoto, T. Ogawa E. Ishida, H. Okada, K. Sakaguchi} Department of Gastroenterology and Hepatology, Okayama University Graduate School of Medicine, Dentistry, and Pharmaceutical Sciences, Okayama, Japan. 


\section{UCTN}

\section{References}

${ }^{1}$ Hartel M, Wente MN, Sido B et al. Carcinoid of the ampulla of Vater. J Gastroenterol Hepatol 2005; 20: 676-681

2 Makhlouf HR, Burke AP, Sobin LH. Carcinoid tumors of the ampulla of Vater: a comparison with duodenal carcinoid tumors. Cancer 1999; 85: 1241 - 1249
${ }^{3}$ Hatzitheoklitos E, Buchler MW, Friess $\mathrm{H}$ et al. Carcinoid of the ampulla of Vater: clinical characteristics and morphologic features. Cancer 1994; 73: $1580-1588$

${ }^{4}$ Ricci JL. Carcinoid of the ampulla of Vater: local resection or pancreaticoduodenectomy. Cancer 1993; 71: 686-690
Corresponding author

\section{H. Kawamoto, MD}

Department of Gastroenterology and Hepatology

Okayama University Graduate School of Medicine, Dentistry, and Pharmaceutical Sciences

2-5-1 Shikata-cho

Okayama 700-8558

Japan

Fax: $\quad$ +81-86-223-5991

Email: h-kawamo@md.okayama-u.ac.jp 